\title{
Real-time tracking of self-reported symptoms to predict potential COVID-19
}

\author{
Cristina Menni ${ }^{1,7 凶}$, Ana M. Valdes ${ }^{1}{ }^{1,2,7}$, Maxim B. Freidin ${ }^{\circledR 1}$, Carole H. Sudre ${ }^{3}$, Long H. Nguyen ${ }^{4}{ }^{4}$, \\ David A. Drew ${ }^{4}{ }^{4}$, Sajaysurya Ganesh ${ }^{5}{ }^{5}$, Thomas Varsavsky3 ${ }^{3}$ M. Jorge Cardoso ${ }^{3}$, \\ Julia S. El-Sayed Moustafa ${ }^{1}{ }^{1}$, Alessia Visconti', Pirro Hysi', Ruth C. E. Bowyer ${ }^{1}{ }^{1}$, Massimo Mangino ${ }^{1,6}$, \\ Mario Falchi', Jonathan Wolf ${ }^{5}$, Sebastien Ourselin ${ }^{3}$, Andrew T. Chan ${ }^{4}$, Claire J. Steves ${ }^{1,8}$ \\ and Tim D. Spector ${ }^{1,8} \bowtie$
}

\begin{abstract}
A total of $2,618,862$ participants reported their potential symptoms of COVID-19 on a smartphone-based app. Among the 18,401 who had undergone a SARS-CoV-2 test, the proportion of participants who reported loss of smell and taste was higher in those with a positive test result $(4,668$ of 7,178 individuals; $65.03 \%$ ) than in those with a negative test result $(2,436$ of 11,223 participants; $21.71 \%$ ) (odds ratio $=6.74$; $95 \%$ confidence interval $=6.31-7.21$ ). A model combining symptoms to predict probable infection was applied to the data from all app users who reported symptoms $(805,753)$ and predicted that $140,312(17.42 \%)$ participants are likely to have COVID-19.
\end{abstract}

COVID-19 is an acute respiratory illness caused by the novel coronavirus severe acute respiratory syndrome coronavirus 2 (SARS-CoV-2). Since its outbreak in China in December 2019, over 2,573,143 cases have been confirmed worldwide (as of 21 April 2020; https://www.worldometers.info/coronavirus/). Although many people have presented with flu-like symptoms, widespread population testing is not yet available in most countries, including the United States (https://www.cdc.gov/coronavirus/2019-ncov/ cases-updates/testing-in-us.html) and United Kingdom ${ }^{1}$. Thus, it is important to identify the combination of symptoms most predictive of COVID-19, to help guide recommendations for self-isolation and prevent further spread of the disease ${ }^{2}$.

Case reports and mainstream media articles from various countries indicate that a number of patients with diagnosed COVID-19 developed anosmia (loss of smell) $)^{3,4}$. Mechanisms of action for the SARS-CoV-2 viral infection causing anosmia have been postulated ${ }^{5,6}$. Other studies indicate that a number of infected individuals present anosmia in the absence of other symptoms ${ }^{7,8}$, suggesting that this symptom could be used as screening tool to help identify people with potential mild cases who could be recommended to self-isolate .

We investigated whether loss of smell and taste is specific to COVID-19 in 2,618,862 individuals who used an app-based symptom tracker ${ }^{10}$ (Methods). The symptom tracker is a free smartphone application that was launched in the United Kingdom on 24 March 2020, and in the United States on 29 March 2020. It collects data from both asymptomatic and symptomatic individu- als and tracks in real time how the disease progresses by recording self-reported health information on a daily basis, including symptoms, hospitalization, reverse-transcription PCR (RT-PCR) test outcomes, demographic information and pre-existing medical conditions.

Between 24 March and 21 April 2020, 2,450,569 UK and 168,293 US individuals reported symptoms through the smartphone app. Of the 2,450,569 participants in the United Kingdom, 789,083 (32.2\%) indicated having one or more potential symptoms of COVID-19 (Table 1). In total, 15,638 UK and 2,763 US app users reported having had an RT-PCR SARS-CoV-2 test, and having received the outcome of the test. In the UK cohort, 6,452 participants reported a positive test and 9,186 participants had a negative test. In the cohort from the United Kingdom, of the 6,452 participants who tested positive for SARS-CoV-2, 4,178 (64.76\%) reported loss of smell and taste, compared with 2,083 out of 9,186 participants $(22.68 \%)$ who tested negative (odds ratio $(\mathrm{OR})=6.40 ; 95 \%$ confidence interval $(C I)=5.96-6.87 ; P<0.0001$ after adjusting for age, sex and body mass index (BMI)). We replicated this result in the US subset of participants who had been tested for SARS-CoV-2 (adjusted $\mathrm{OR}=10.01 ; 95 \% \mathrm{CI}=8.23-12.16 ; P<0.0001)$ and combined the adjusted results using inverse variance fixed-effects meta-analysis $(\mathrm{OR}=6.74 ; 95 \% \mathrm{CI}=6.31-7.21 ; P<0.0001)$.

We re-ran logistic regressions adjusting for age, sex and BMI to identify other symptoms besides anosmia that might be associated with being infected by SARS-CoV-2. All ten symptoms queried (fever, persistent cough, fatigue, shortness of breath, diarrhea, delirium, skipped meals, abdominal pain, chest pain and hoarse voice) were associated with testing positive for COVID-19 in the UK cohort, after adjusting for multiple testing (Fig. 1a). In the US cohort, only loss of smell and taste, fatigue and skipped meals were associated with a positive test result.

We performed stepwise logistic regression in the UK cohort, by randomlydividing it into training and test sets (ratio: 80:20) to identify independent symptoms most strongly correlated with COVID-19, adjusting for age, sex and BMI. A combination of loss of smell and taste, fatigue, persistent cough and loss of appetite resulted in the best model (with the lowest Akaike information criterion). We therefore generated a linear model for symptoms that included loss

'Department of Twin Research and Genetic Epidemiology, King's College London, London, UK. ${ }^{2}$ Academic Rheumatology, Clinical Sciences, Nottingham City Hospital, Nottingham, UK. ${ }^{3}$ School of Biomedical Engineering \& Imaging Sciences, King's College London, London, UK. ${ }^{4}$ Clinical and Translational Epidemiology Unit, Massachusetts General Hospital and Harvard Medical School, Boston, MA, USA. ${ }^{5}$ Zoe Global, London, UK. ${ }^{6} \mathrm{NIHR}$ Biomedical Research Centre at Guy's and St Thomas' Foundation Trust, London, UK. ${ }^{7}$ These authors contributed equally: Cristina Menni, Ana M. Valdes. ${ }^{8}$ These authors jointly

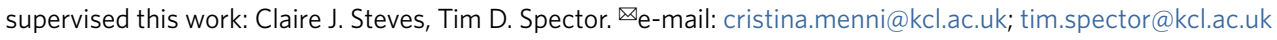


Table 1 | Characteristics of the study population

\begin{tabular}{|c|c|c|c|c|c|c|}
\hline & \multicolumn{4}{|c|}{ Tested for SARS-CoV-2 } & \multicolumn{2}{|c|}{ Not tested for SARS-CoV-2 } \\
\hline & \multicolumn{2}{|l|}{ United Kingdom } & \multicolumn{2}{|l|}{ United States } & \multirow[t]{2}{*}{ United Kingdom } & \multirow[t]{2}{*}{ United States } \\
\hline & Tested positive & Tested negative & Tested positive & Tested negative & & \\
\hline Female (\%) & 71.88 & 76.40 & 78.10 & 82.28 & 64.24 & 68.67 \\
\hline Age (years) & $41.25(12.18)$ & $41.87(12.14)$ & $44.65(14.31)$ & $47.25(13.80)$ & $43.38(14.69)$ & $53.00(16.68)$ \\
\hline BMI $\left(\mathrm{kg} \mathrm{m}^{-2}\right)$ & $27.34(6.06)$ & $27.04(5.91)$ & $28.04(6.91)$ & $27.71(636)$ & $26.39(5.44)$ & $27.08(5.87)$ \\
\hline $\begin{array}{l}\text { Loss of smell and taste } \\
\text { (\%) }\end{array}$ & 64.76 & 22.68 & 67.49 & 17.33 & 9.50 & 12.10 \\
\hline Fatigue (\%) & 29.80 & 15.56 & 23.42 & 21.50 & 8.71 & 8.17 \\
\hline Shortness of breath (\%) & 15.27 & 9.37 & 13.50 & 12.03 & 4.52 & 3.65 \\
\hline Delirium (\%) & 17.87 & 13.25 & 23.55 & 23.47 & 15.26 & 16.25 \\
\hline Skipped meals (\%) & 42.03 & 24.93 & 47.25 & 36.08 & 19.12 & 21.42 \\
\hline Abdominal pain (\%) & 21.31 & 18.24 & 20.94 & 21.06 & 3.70 & 15.94 \\
\hline Chest pain (\%) & 42.73 & 39.17 & 38.57 & 41.92 & 18.65 & 24.74 \\
\hline Hoarse voice (\%) & 31.70 & 25.85 & 26.86 & 25.72 & 7.86 & 13.79 \\
\hline
\end{tabular}

The results are presented as percentage values for dichotomous traits and as means and standard deviation (in parentheses) for continuous traits (age and BMI).

of smell and taste, fatigue, persistent cough and loss of appetite to obtain a symptoms prediction model for COVID-19:

Prediction model $=-1.32-(0.01 \times$ age $)$

$+(0.44 \times \operatorname{sex})+(1.75 \times$ loss of smell and taste $)$

$+(0.31 \times$ severe or significant persistent cough $)$

$+(0.49 \times$ severe fatigue $)+(0.39 \times$ skipped meals $)$

where all symptoms are coded as 1 if the person self-reports the symptom and 0 if not. The sex feature is also binary, with 1 indicative of male participants and 0 representing females. The obtained value is then transformed into predicted probability using $\exp (x) /$ $(1+\exp (x))$ transformation followed by assigning cases of predicted COVID-19 for probabilities $>0.5$ and controls for probabilities $<0.5$.

In the UK test set, the prediction model had a sensitivity of $0.65(0.62-0.67)$, a specificity of $0.78(0.76-0.80)$, an area under the curve (AUC) of the receiver operating characteristic curve (ROC) (that is, ROC-AUC) of $0.76(0.74-0.78)$, a positive predictive value of $0.69(0.66-0.71)$ and a negative predictive value of 0.75 (0.73-0.77) (Fig. 1b). A cross-validation ROC-AUC was 0.75 (0.74$0.76)$ in the 15,638 UK users who were tested for SARS-CoV-2. In this model, the strongest predictor was loss of smell and taste (Fig. 1a). Excluding loss of smell and taste from the model resulted in reduced sensitivity $(0.33(0.30-0.35))$ but increased specificity (0.84 (0.83-0.86)). We also computed the ROC-AUC with stratification for sex and age groups and found that the results were similar in all groups, with no significant differences between strata, suggesting that our model works similarly within different sex and age groups. We validated the model in the US cohort and found an ROC-AUC of $0.76(0.74-0.78)$, a sensitivity of $0.66(0.62-0.69)$, a specificity of $0.83(0.82-0.85)$, a positive predictive value of $0.58(0.55-0.62)$ and a negative predictive value 0.87 (0.86-0.89) (Fig. 1c).

We also queried whether the association between loss of smell and taste and COVID-19 was influenced by mainstream media reports. We assessed the correlation between loss of smell and taste and being COVID-19 positive in different date ranges: (1) 24 March to 3 April 2020, following a number of reports in the UK mainstream media (for example, ref. ${ }^{11}$ ) reporting anosmia as a symptom of COVID-19; (2) the week of 4-10 April 2020; and (3) from 11-21 April 2020. In the United Kingdom, the OR (95\% CI) values for the associations of self-reported loss of smell and taste and a positive test for COVID-19 across these periods were 4.98 (4.47-5.56), 6.64 (5.75-7.68) and 10.40 (9.08-11.91), respectively, suggesting that awareness of loss of smell and taste as symptoms of COVID19 in the UK has increased following media reports. However, this association was not found in the US cohorts: 24 March to 3 April: 8.13 (5.18-12.78); 4-10 April: 12.30 (8.96-16.90); 11-21 April: 9.13 (6.73-12.38).

Finally, we applied the predictive model to the 805,753 UK and US symptom-reporting individuals who had not been tested for COVID-19 and found that, according to our model, 140,312 $(116,400-164,224)$ of these 805,753 participants $(17.42 \%$ (14.45$20.39 \%)$ ) reporting some symptoms were likely to be infected by the virus, representing $5.36 \%$ as a proportion of the overall responders to the app.

We report that loss of smell and taste is a potential predictor of COVID-19 in addition to other, more established, symptoms including high temperature and a new, persistent cough. COVID19 appears to cause problems of smell receptors in line with many other respiratory viruses, including previous coronaviruses thought to account for $10-15 \%$ of cases of anosmia ${ }^{7,9}$.

We also identify a combination of symptoms, including anosmia, fatigue, persistent cough and loss of appetite, that together might identify individuals with COVID-19.

A major limitation of the current study is the self-report nature of the data included, which cannot replace physiological assessments of olfactory and gustatory function or nucleotide-based testing for SARS-CoV-2. Both false negative and false positive 

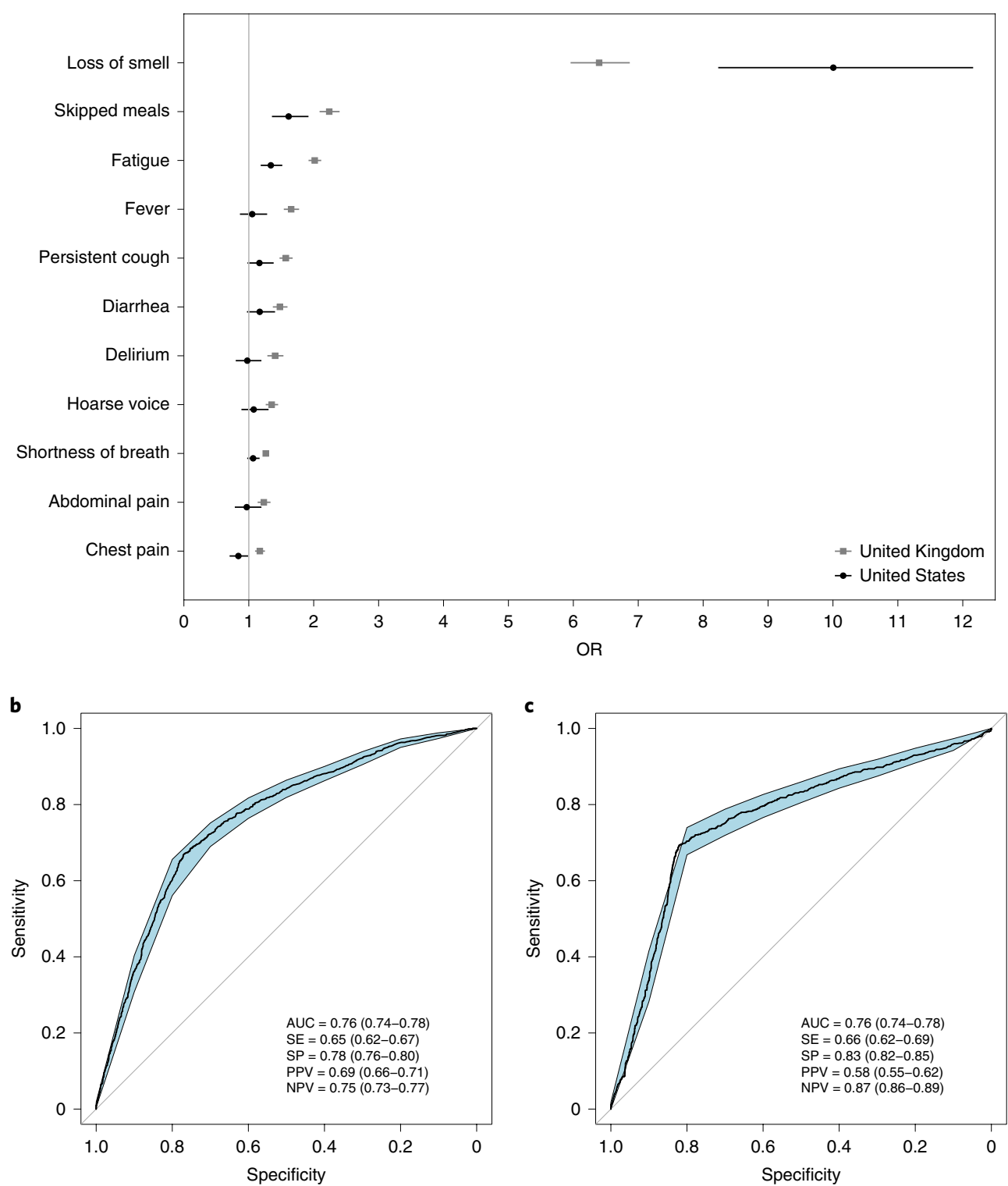

Fig. 1 | Association between symptoms and SARS-CoV-2 infection, and ROCs for prediction of the risk of a positive test. a, Association between symptoms and the odds ratio of SARS-CoV-2 infection in 15,638 UK and 2,763 US participants who were tested via RT-PCR. Error bars represent 95\% $\mathrm{CIs}$. b,c, ROCs for prediction in the UK test set (b) and US validation set (c) of the risk of a positive test for SARS-CoV-2, using the following self-reported symptoms and traits: persistent cough, fatigue, skipped meals, loss of smell and taste, sex and age. Values for AUC, sensitivity (SE), specificity (SP), positive predictive value (PPV) and negative predictive value (NPV) are shown, with $95 \% \mathrm{Cl}$ in parentheses.

reports could be included in the dataset ${ }^{12}$, and because of the way the questions are asked, gustatory and olfactory losses are conflated. Second, at present, we do not know whether anosmia was acquired before or after other COVID-19 symptoms, or during the illness or afterwards. This information could become available as currently healthy users track symptom development over time. As more accurate tests become available, we have the ability to optimize our model. One caveat of our study is that the individuals on which the model was trained are not representative of the general population because performing tests for SARS-CoV-2 is not random. Testing is more likely to be done if an individual develops severe symptoms requiring hospitalization, if an individual has been known to have had contact with people who have tested positive for SARS-CoV-2 infection, in health workers, and if an individual has traveled in an area of high risk of exposure. Therefore, our results may overestimate the number of expected positive cases of SARS-CoV-2 infec- tion. Additionally, volunteers using the app are a self-selected group who might not be fully representative of the general population. Another limitation is the potential effect that mainstream media coverage of loss of smell and taste and COVID-19 might have had on app responses. We found that these reports might have influenced UK responders, for whom there was a temporal trend in the strength of the association. However, there was no such association in the US cohort; therefore, we conclude that regardless of any bias introduced by mainstream media reports, the association between COVID-19 and loss of smell and taste remains strong.

Our work suggests that loss of sense of smell and taste could be included as part of routine screening for COVID-19 and should be added to the symptom list currently developed by the World Health Organization (www.who.int/health-topics/coronavirus). A detailed study on the natural history of broader COVID-19 symptoms, especially according to timing and frequency, will help us to understand 
the usefulness of symptom tracking and modeling, and to identify probable clusters of infection.

\section{Online content}

Any methods, additional references, Nature Research reporting summaries, source data, extended data, supplementary information, acknowledgements, peer review information; details of author contributions and competing interests; and statements of data and code availability are available at https://doi.org/10.1038/s41591020-0916-2.

Received: 10 April 2020; Accepted: 30 April 2020;

Published online: 11 May 2020

\section{References}

1. Whittington, A. M. et al. Coronavirus: rolling out community testing for COVID-19 in the NHS. BMJ Opinion https://blogs.bmj.com/bmj/2020/02/17/ coronavirus-rolling-out-community-testing-for-covid-19-in-the-nhs/ (2020).

2. Rossman, H. et al. A framework for identifying regional outbreak and spread of COVID-19 from one-minute population-wide surveys. Nat. Med. https:// doi.org/10.1038/s41591-020-0857-9 (2020)

3. Gane, S. B., Kelly, C. \& Hopkins, C. Isolated sudden onset anosmia in COVID-19 infection. A novel syndrome? Rhinology https://doi.org/10.4193/ Rhin20.114 (2020).

4. Iacobucci, G. Sixty seconds on... anosmia. Br. Med. J. 368, m1202 (2020).
5. Brann, D. et al. Non-neuronal expression of SARS-CoV-2 entry genes in the olfactory system suggests mechanisms underlying COVID-19-associated anosmia. Preprint at bioRxiv https://www.biorxiv.org/content/10.1101/2020.03 $25.009084 \mathrm{v} 3(2020)$

6. Sungnak, W. et al. SARS-CoV-2 entry factors are highly expressed in nasal epithelial cells together with innate immune genes. Nat. Med. https://doi. org/10.1038/s41591-020-0868-6 (2020).

7. Hopkiins, C. \& Kumar, N. Loss of Sense of Smell as Marker of COVID-19 Infection (ENT UK, 2020).

8. Eliezer, M. et al. Sudden and complete olfactory loss function as a possible symptom of COVID-19. JAMA Otolaryngol. Head Neck Surg. https://doi. org/10.1001/jamaoto.2020.0832 (2020).

9. Spinato, G. et al. Alterations in smell or taste in mildly symptomatic outpatients with SARS-CoV-2 infection. J. Am. Med. Assoc. https://doi. org/10.1001/jama.2020.6771 (2020).

10. Drew, D. et al. Rapid implementation of mobile technology for real-time epidemiology of COVID-19. Science https://science.sciencemag.org/content/ early/2020/05/04/science.abc0473/tab-article-info (2020).

11. Roberts, M. Coronavirus: are loss of smell and taste key symptoms? $B B C$ News (1 April 2020).

12. Oleszkiewicz, A., Kunkel, F., Larsson, M. \& Hummel, T. Consequences of undetected olfactory loss for human chemosensory communication and well-being. Phil. Trans. R. Soc. Lond. B Biol. Sci. 375, 20190265 (2020).

Publisher's note Springer Nature remains neutral with regard to jurisdictional claims in published maps and institutional affiliations.

() The Author(s), under exclusive licence to Springer Nature America, Inc. 2020 


\section{Methods}

Study setting and participants. The COVID Symptom Study smartphone-based app (previously known as COVID Symptom Tracker) was developed by Zoe Global, in collaboration with King's College London and Massachusetts General Hospital, and was launched in the United Kingdom on 24 March 2020 and in the United States on 29 March 2020. After 3 weeks, it had reached 2,618,862 users. It enables the capture of self-reported information related to COVID-19, as described previously ${ }^{10}$. The survey questions are available in Supplementary Table 1. On first use, the app records self-reported location, age and core health risk factors. With continued use and notifications, participants provide daily updates on symptoms, health care visits, COVID-19 testing results and whether they are self-quarantining or seeking health care, including the level of intervention and related outcomes. Individuals without apparent symptoms are also encouraged to use the app.

Ethics. The King's College London Ethics Committee approved the ethics for the app, and all users provided consent for non-commercial use. An informal consultation with TwinsUK members over email and social media before the app was launched found that they were overwhelmingly supportive of the project. The US protocol was approved by the Partners Human Research Committee.

Statistical analysis. Data from the app were downloaded to a server and only records where the self-reported characteristics fell within the following ranges were utilized for further analysis: age: 16-90 years (18 years in the United States); height: $110-220 \mathrm{~cm}$; weight: $40-200 \mathrm{~kg}$; BMI: $14-45 \mathrm{~kg} \mathrm{~m}^{-2}$; and temperature: $35-42^{\circ} \mathrm{C}$. The individuals whose data were included to develop and test the prediction model were those who had completed the report for symptoms in the app and who declared that they had been tested for SARS-CoV-2 by RT-PCR and received the result. Only individuals who answered at least nine of the ten symptom questions, and who answered about loss of smell and taste, were included.

Baseline characteristics are presented as the number (percentage) for categorical variables and the mean (standard deviation) for continuous variables. Multivariate logistic regression adjusting for age, sex and BMI was applied to investigate the correlation between loss of smell and taste and COVID-19 in 15,368 UK users of the symptom tracker app who were also tested in the laboratory for SARS-CoV-2 (6,452 UK individuals tested positive and 9,186 tested negative). The results were replicated in 726 US individuals who tested positive and 2,037 US individuals who tested negative. We then randomly split the UK sample into training and test sets with a ratio of 80:20. In the training set, we performed stepwise logistic regression combining forward and backward algorithms, to identify other symptoms associated with COVID-19 independent of loss of smell and taste. We included in the model ten other symptoms (fever, persistent cough, fatigue, shortness of breath, diarrhea, delirium, skipped meals, abdominal pain, chest pain and hoarse voice) as well as age, sex and BMI, and chose as the best model the one with the lowest Akaike information criterion. We then assessed the performance of the model both in the test set and via tenfold cross-validation in the entire UK sample of 15,638 individuals using the R package $\mathrm{cvAUC}^{13}$. We further validated the prediction model in the US cohort.

For our predictive model, using the R packages $\mathrm{pROC}$ and epiR, we further computed the AUC (that is, the overall diagnostic performance of the model), sensitivity (positivity in disease; that is, the proportion of subjects who have the target condition (reference standard positive) and give positive test results) and specificity (negativity in health; that is, the proportion of subjects without a SARS-CoV-2 RT-PCR test who give negative model results).

Finally, we applied the predictive model to the 805,753 individuals reporting symptoms who had not had a SARS-CoV-2 test, to estimate the percentage of individuals reporting some COVID-19 symptoms who were likely to be infected by the virus. The proportion of estimated infections was calculated repeatedly by sampling the dataset (with replacement) to obtain the $95 \%$ CIs.

Reporting Summary. Further information on research design is available in the Nature Research Reporting Summary linked to this article.

\section{Data availability}

Data collected in the app are being shared with other health researchers through the NHS-funded Health Data Research UK (HDRUK)/SAIL consortium, housed in the UK Secure e-Research Platform (UKSeRP) in Swansea. Anonymized data collected by the symptom tracker app can be shared with bonafide researchers via HDRUK, provided the request is made according to their protocols and is in the public interest (see https://healthdatagateway.org/detail/9b604483$9 \mathrm{cdc}-41 \mathrm{~b} 2-\mathrm{b} 82 \mathrm{c}-14 \mathrm{ee} 3 \mathrm{dd} 705 \mathrm{f} 6)$. US investigators are encouraged to coordinate data requests through the COPE Consortium (www.monganinstitute.org/ cope-consortium). Data updates can be found at https://covid.joinzoe.com.

\section{Code availability}

The app code is publicly available from https://github.com/zoe/

covid-tracker-react-native.

\section{References}

13. LeDell, E., Petersen, M. \& van der Laan, M. Computationally efficient confidence intervals for cross-validated area under the ROC curve estimates. Electron J. Stat. 9, 1583-1607 (2015).

\section{Acknowledgements}

We express our sincere thanks to all of the participants who entered data into the app, including study volunteers enrolled in cohorts within the Coronavirus Pandemic Epidemiology (COPE) consortium. We thank the staff of Zoe Global, the Department of Twin Research at King's College London and the Clinical and Translational Epidemiology Unit at Massachusetts General Hospital for tireless work in contributing to the running of the study and data collection. We thank E. Segal and his laboratory for helpful input. This work was supported by Zoe Global. The Department of Twin Research receives grants from the Wellcome Trust (212904/Z/18/Z) and Medical Research Council/British Heart Foundation Ancestry and Biological Informative Markers for Stratification of Hypertension (AIMHY; MR/M016560/1), and support from the European Union, the Chronic Disease Research Foundation, Zoe Global, the NIHR Clinical Research Facility and the Biomedical Research Centre (based at Guy's and St Thomas' NHS Foundation Trust in partnership with King's College London). C.M. is funded by the Chronic Disease Research Foundation and by the Medical Research Council AIM HY project grant. A.M.V. is supported by the National Institute for Health Research Nottingham Biomedical Research Centre. C.H.S. received an Alzheimer's Society Junior Fellowship (AS-JF-17-011). S.O. and M.J.C. are funded by the Wellcome/ EPSRC Centre for Medical Engineering (WT203148/Z/16/Z) and Wellcome Flagship Programme (WT213038/Z/18/Z). A.T.C. is the Stuart and Suzanne Steele MGH Research Scholar and is a team leader for the Stand Up to Cancer Foundation. A.T.C., L.H.N. and D.A.D. are supported by an Evergrande COVID-19 Response Fund Award through the Massachusetts Consortium on Pathogen Readiness (MassCPR). This work was also supported by the UK Research and Innovation London Medical Imaging \& Artificial Intelligence Centre for Value-Based Healthcare.

\section{Author contributions}

C.M., A.M.V., J.W., C.J.S. and T.D.S. conceived of and designed the experiments. C.M., M.B.F., C.H.S., S.G., and A.M.V. analyzed the data. T.V., S.O., M.J.C., R.C.E.B., A.V., J.S.E.S.-M., P.H., M.M., M.F., D.A.D., A.T.C. and L.H.N. contributed reagents, materials and/or analysis tools. C.M. and A.M.V. wrote the manuscript. All authors revised the manuscript.

\section{Competing interests}

T.D.S. and A.M.V. are consultants to Zoe Global. S.G. and J.W. are employees of Zoe Global.

\section{Additional information}

Supplementary information is available for this paper at https://doi.org/10.1038/ s41591-020-0916-2.

Correspondence and requests for materials should be addressed to C.M. or T.D.S. Peer review information Jennifer Sargent was the primary editor on this article and managed its editorial process and peer review in collaboration with the rest of the editorial team.

Reprints and permissions information is available at www.nature.com/reprints. 


\section{natureresearch}

Corresponding author(s): Cristina Menni

Last updated by author(s): 29/04/2020

\section{Reporting Summary}

Nature Research wishes to improve the reproducibility of the work that we publish. This form provides structure for consistency and transparency in reporting. For further information on Nature Research policies, see Authors \& Referees and the Editorial Policy Checklist.

\section{Statistics}

For all statistical analyses, confirm that the following items are present in the figure legend, table legend, main text, or Methods section.

n/a Confirmed

$\bigotimes$ The exact sample size $(n)$ for each experimental group/condition, given as a discrete number and unit of measurement

$\bigotimes$ A statement on whether measurements were taken from distinct samples or whether the same sample was measured repeatedly

The statistical test(s) used AND whether they are one- or two-sided

Only common tests should be described solely by name; describe more complex techniques in the Methods section.

$\bigotimes$ A description of all covariates tested

$\square$ \A description of any assumptions or corrections, such as tests of normality and adjustment for multiple comparisons

$\triangle$ A full description of the statistical parameters including central tendency (e.g. means) or other basic estimates (e.g. regression coefficient)

AND variation (e.g. standard deviation) or associated estimates of uncertainty (e.g. confidence intervals)

For null hypothesis testing, the test statistic (e.g. $F, t, r$ ) with confidence intervals, effect sizes, degrees of freedom and $P$ value noted

Give $P$ values as exact values whenever suitable.

\ $\square$ For Bayesian analysis, information on the choice of priors and Markov chain Monte Carlo settings

$\square$ \ For hierarchical and complex designs, identification of the appropriate level for tests and full reporting of outcomes

$\square \bigotimes$ Estimates of effect sizes (e.g. Cohen's $d$, Pearson's $r$ ), indicating how they were calculated

Our web collection on statistics for biologists contains articles on many of the points above.

\section{Software and code}

Policy information about availability of computer code

Data collection The COVID Symptom Tracker smartphone based app (version 0.13) was developed by Zoe Global Limited, King's College London, and Massachusetts General Hospital, and was launched in the UK on Tuesday the 24th March 2020, and in the US on 29th March 2020. On first use, the app records self-reported location, age, and core health risk factors. With continued use and notifications, participants provide daily updates on symptoms, health care visits, COVID-19 testing results, and if they are self-quarantining or seeking health care, including the level of intervention and related outcomes. Individuals without apparent symptoms are also encouraged to use the app. Data from the app is automatically downloaded into a protected server.

Data analysis

Analyses were carried out using version 3.6.0. The prediction model was fitted using standard R functions. The R package epiR 1.0-14, was used to calculate its performance statistics (SE, SP, PPV, NPV), while the receiver operating characteristic (ROC) curves were compared with the R package PROC 1.16.2.

For manuscripts utilizing custom algorithms or software that are central to the research but not yet described in published literature, software must be made available to editors/reviewers. We strongly encourage code deposition in a community repository (e.g. GitHub). See the Nature Research guidelines for submitting code \& software for further information.

\section{Data}

Policy information about availability of data

All manuscripts must include a data availability statement. This statement should provide the following information, where applicable:

- Accession codes, unique identifiers, or web links for publicly available datasets

- A list of figures that have associated raw data

- A description of any restrictions on data availability

Data collected in the app is being shared with other health researchers through the NHS funded Health Data Research UK (HDRUK)/SAIL consortium, housed in the UK Secure Research Platform (UKSeRP) in Swansea. Anonymised data is available to be shared with bonafide researchers HDRUK according to their protocols in the public interest. See https://healthdatagateway.org/detail/9b604483-9cdc-41b2-b82c-14ee3dd705f6.

US investigators are encouraged to coordinate data requests through the COPE Consortium (www.monganinstitute.org/cope-consortium). Data updates can be 


\section{Field-specific reporting}

Please select the one below that is the best fit for your research. If you are not sure, read the appropriate sections before making your selection. \Life sciences Behavioural \& social sciences Ecological, evolutionary \& environmental sciences

For a reference copy of the document with all sections, see nature.com/documents/nr-reporting-summary-flat.pdf

\section{Life sciences study design}

All studies must disclose on these points even when the disclosure is negative.

Sample size

Given the observation nature of our study, we did not perform statistical analyses to predetermine sample size.

Between March 24, 2020 and April 21, 2020, 2,450,569 UK and 168,293 US individuals reported symptoms through the smartphone app. 15,638 UK and 2,763 US app users reported having had an RT-PCR SARS-CoV-2 test, and having received the outcome of the test $(6,452$ UK individual tested positive and 9,186 tested negative; 726 US individual tested positive and 2,037 US individual tested negative). All the 18,401 who had undergone a SARS-CoV-2 test, were included to test the association between loss of smell and taste and been tested positive and also were included to develop and test the prediction model. The prediction model was then applied to all app users.

Data exclusions The individuals whose data was included to develop and test the prediction model were those who completed the report for symptoms in the app, who declared to have had a SARS-CoV-2 RT-PCR test and to have received the result for the test. Only individuals who answered at least 9 of the 10 symptoms and answered about loss of smell and taste were included. The data exclusion criteria for the prediction phase were pre-established. The prediction model was then applied to all app users.

Replication The association between loss of smell and taste and testing positive for COVID-19 was successfully replicated in the US cohort as well as the prediction model.

Randomization Our study is an observational study so no randomization is needed here.

Blinding This is not relevant given the observational nature of our study

\section{Reporting for specific materials, systems and methods}

We require information from authors about some types of materials, experimental systems and methods used in many studies. Here, indicate whether each material, system or method listed is relevant to your study. If you are not sure if a list item applies to your research, read the appropriate section before selecting a response.

Materials \& experimental systems

Methods

$\mathrm{n} / \mathrm{a}$ Involved in the study

Х $\square$ Antibodies

$\bigotimes \square$ Eukaryotic cell lines

\ $\square$ Palaeontology

$\mathrm{n} / \mathrm{a}$ Involved in the study

\ $\square$ ChIP-seq

\ $\square$ Flow cytometry

$\triangle \square$ Animals and other organisms

\ $\square$ MRI-based neuroimaging

$\square \bigotimes$ Human research participants

Х $\square$ Clinical data

\section{Human research participants}

Policy information about studies involving human research participants

Population characteristics Study participants are 2,450,569 UK and 168,293 US adult users of the COVID Symptom Tracker, a smartphone app. Of the 2,450,569 participants in the UK, 789,083 (32.2\%) indicated having one or more potential symptoms of COVID-19. 15,638 UK and 2,763 US app users reported having had an RT-PCR SARS-CoV-2 test, and having received the outcome of the test.

Users are $65 \%$ females, are slightly overweight and have a mean age of 44 yrs with a range from 18 to 90 years. All users are able to provide written informed consent.

Recruitment

The COVID Symptom Tracker smartphone based app was developed by Zoe Global Limited (a digital healthcare company), King's College London, and Massachusetts General Hospital. By leveraging the established digital backbone of an application used for personal nutrition studies, the COVID Symptom Tracker was launched in the UK on Tuesday the 24th March 2020, and in the US on 29th March 2020 and after three weeks has reached 2,618,862 users. Volunteers using the app are a self-selected group who might not be fully representative of the general population. 
